\title{
A survey and analysis based on image denoising method
}

\author{
Abhishek Dipak Shroff ${ }^{1 *}$, Kailash Patidar ${ }^{2}$ and Rishi Kushwah ${ }^{3}$ \\ M.Tech Scholar, Department of Computer Science, School of Engineering, Sri Satya Sai University of Technology \\ $\&$ Medical Sciences, Sehore, Madhya Pradesh, India ${ }^{1}$ \\ Professor and HOD, Department of Computer Science, School of Engineering, Sri Satya Sai University of \\ Technology \& Medical Sciences, Sehore, Madhya Pradesh, India ${ }^{2}$ \\ Assistant Professor, Department of Computer Science, School of Engineering, Sri Satya Sai University of \\ Technology \& Medical Sciences, Sehore, Madhya Pradesh, India ${ }^{3}$
}

\section{(C)2018 ACCENTS}

\begin{abstract}
There are different methods have been presented for the noise reduction. It is essential in proper formation and retrieval of images. This paper explores the methods previously proposed. There are several research work is in progress in the process of denoising. Still there are lot of scope in this field for the betterment. This paper elaborates the previous methods along with the gap found and focus on the advantages. This discussion is based on the latest literature included in this paper. This paper also provides the meta-analysis and comparative discussion of different denoising methods. Based on the gaps identified future suggestions have been listed.
\end{abstract}

\section{Keywords}

Image denoising, Image retrieval, Noise reduction, Denoising methods.

\section{Introduction}

In the current scenario image denoising in the trending research area. As it is used in different areas of research work including medical image analysis for the purpose of diagnosis.

Correspondence of computerized pictures through the remote sensors or on the other hand organizes turned into a costly errand, because of the misfortune of data in the correspondence way that outcomes in poor remaking. By and large, remaking of a flag is finished by Shannon examining hypothesis. It expresses that reproduction is conceivable if and just if the flag is transmitted with Nyquist rate [1-3]. On account of pictures, the number of tests is high. Transmission of those numerous examples with twofold information rate is a mind boggling errand and elucidation of tests requires exceptionally designed machines [4].

All these factors make computerized picture correspondence as unpredictable and costly correspondence frameworks [5].

*Author for correspondence
According to Nyquist/Shannon inspecting hypothesis, the inspecting rate ought to be no less than double the most extreme recurrence exhibit in the flag $[6,7]$. Compacted detecting permits as to test the information well underneath the Nyquist rate. Truth be told, compacted detecting hypothesis empowers a 4-overlap lessening in obtaining time for biomedical pictures by permitting the undersampling [8]. It has numerous applications in various fields, including medical imaging, seismic imaging, dispersed and remote detecting, analog to digital conversion [9-11].

Along these lines, it can successfully decorrelate the conditions found over picture discontinuities and minimal high recurrence parts incited by picture highlights into the lower level or low band pass.

In the event that we consider the wavelet change then it can be adequately catch particular indicates up two measurements implies including one measurement, yet it is flop in speaking to the real highlights like edge , shading, shape et cetera. There are a few directional and non-directional repetitive changes which are investigated in various research papers, including the curvelet, contourlet, wedgelet, bandlet, and the steerable wavelet [12-14]. 
There are a few methodologies which are fundamentally worry with denoise a picture information, for example, averaging channel, Median channel, Gaussian channel and partial differential equations (PDE) approach. On the off chance that we investigate the properties of good pictures then it will be with the fewer clamors and limit the obscure or obscure lessening is the imperative factor. The PDE approach is much viable and applies in a few researches like $[15,16]$. Be that as it may, it is more powerful on the off chance that we apply fourth request fractional differential condition. Utilizations of the PDE models can be generally found in a wide scope of picture reclamation errands, for example, denoising and improvement [17] shading picture handling and determination. This gives us the future knowledge or work with the forward request fractional differential condition with a similar request toward obscure decrease.

\section{Related work}

In 2011, Lang et al. [18] proposed a joined transform picture denoising calculation in view of morphological component analysis (MCA). They have suggested that the approach is helpful in the image separation into natural scene and linear singular structure. Curvelet change limit denosing is utilized as a part of straight particular structure while wavelet change manages smooth part. This calculation makes full utilization of individual points of interest of the wavelet change and curvelet change. Investigation comes about demonstrate that the calculation can better keep up the points of interest qualities in managing the picture with straight peculiarity, and it has a superior denosing execution for picture than a basic wavelet thresholding or curvelet thresholding.

In 2012, Su et al. [19] introduced a sparse and redundant combination of algorithm for learned dictionary. It is used to process different types of images. They utilize the K-SVD denoising system and adjust its underlying lexicon, and after that for the most part center on utilizing it to think about its denoising execution and appropriateness for various sorts of Pictures, and after that contrast it and some other picture denoising calculations. With regards to the remote detecting pictures denoising, the analysis comes about demonstrate that the K-SVD calculation can prompts the condition of-craftsmanship denoising execution at low boisterous levels, yet for high loud levels, its execution isn't great on PSNR and visual impact, that is it can't hold the nearby points of interest of pictures.
In 2012, Zhang et al. [20] proposesd an image denoising method which is based on support vector regression. The results show that the denoising is better than the previous method and it is capable in restoring the original image.

In 2017, Chithra and Santhanam [21] proposed a hybrid filter. It is used for suppressing the Gaussian noise in computer tomography (CT) for improving the quality of the image. Their method is the combination of modified median wiener filter (MMWF) and absolute difference and mean (ADMF) filter. It is compared with the triangular and direction-based filter (TDBF), discrete wavelet transform using total variation (DWTTV), edge preserving hybrid filter(EPHF) and ADMF filter. The result shows the proposed method is better in terms of peak signal to noise ratio (PSNR) and mean square error (MSE).

In 2017, Soni and Kirar [22] suggested the challenges found in noise removal. In the course of recent decades, various types of clamor lessening methods have been produced. They audits the change based denoising procedures and plays out their similar examination. They put consequences of various approaches including general ridgelets and curvelets, Experimental Mode Decomposition and Empirical ridgelets and curvelets. A quantitative measure of examinations is displayed as far as PSNR.

In 2017, Pang [23] suggested that the wavelet decomposing levels and the selection of the thresholding function may affect the performance of image denoising. They have presented a new approach for the wavelet decomposing levels identification using the 2D Haar wavelet thresholding method. It utilizes the standard deviation estimations of the sub-groups to see whether the flag vitality is solid or frail in the high recurrence sub-groups after the 2D Haar wavelet change. In expansion, another thresholding capacity is proposed which accomplishes better denoising execution as far as PSNR and MSE than the delicate thresholding technique. Particularly, at high commotion levels, the proposed new thresholding technique outflanks hard thresholding, delicate thresholding and semi-delicate thresholding techniques.

In 2017, Yang and Liu [24] suggested that the Fluorescence molecular imaging tomography (FMT) has the advantages of early intervention, low cost and easy use. FMT can obtain the appropriation territory of fluorophore in body without cutting the body or 
placing something into the body. However, fluorescence sub-atomic picture is extremely delicate to numerous sort of commotions, for example, autofluorescence, background noise a few different commotions. The fluorescence flag is the real segment in fluorescence picture, the power of autofluorescence and other foundation signals are generally low in the analysis result. This technique dispense with high recurrence clamors by Gaussian smoothing in recurrence area, at that point, to extricate the significant segments of the fluorescence motions by wavelet change, at last, $\mathrm{k}$-means grouping is used to isolate the significant part and foundation of the fluorescence picture. Trial comes about demonstrate that the proposed strategy is viability, it could get the fluorescence flag while wipe out the foundation clamor, and increment the nature of fluorescence picture.

In 2017, Ankarao et al. [25] suggested that the orthogonal matching algorithm is cost effective in terms of computational complexity. This calculation gives an answer for over determined also, underdetermined frameworks by limiting the blunder capacities utilizing slightest square. This work focuses on the development of lexicon which can be utilized to comprehend the sparsity based picture denoising issue. They constructed the dictionary least square solution subjected to thresholding conditions Orthogonal matching pursuit (OMP) calculation stays away from the determination of a similar particle in each emphasis, because of the presence of symmetrical property between the deposit and the iota chose from the word reference. Therefore, OMP calculation brings about exact picture reproduction. The proposed strategy is approved on four standard test pictures, for example, Lena,Vessel, Barbara and Cameraman with various clamors, for example, salt and pepper commotion, Gaussian clamor and spot clamor with fluctuating the level of commotion level from $5 \%$ to $40 \%$. Gotten comes about are assessed by the quality metric PSNR and contrasted and the current wavelet based meager picture denoising. The test assessment demonstrates that the proposed technique is better material to expel the dot commotion and salt and pepper clamor when contrasted and the current wavelet based inadequate picture denoising.

\section{Methodological analysis}

The following observations have been analysed based on the previous research and the current trends (Table 1).

Table 1 Methodological analysis

\begin{tabular}{llll}
\hline $\begin{array}{l}\text { S. } \\
\text { No }\end{array}$ & Source & \multicolumn{1}{c}{ Approach } & \multicolumn{1}{c}{ Result } \\
\hline 1 & {$[26]$} & $\begin{array}{l}\text { No-reference image quality } \\
\text { assessment }\end{array}$ & $\begin{array}{l}\text { No-reference image quality assessment (NRIQA) have been used for predict } \\
\text { the quality evaluation and suggested that there is still the chances of } \\
\text { improvement. }\end{array}$ \\
\hline 2 & {$[27]$} & $\begin{array}{l}\text { Boundary discriminated noise } \\
\text { detection (BDND) algorithm }\end{array}$ & $\begin{array}{l}\text { Their Proposed filter is capable in changing the size of the window based on } \\
\text { the noise density computed from noise statistics after the BDND detection. } \\
\text { Their results are better in comparison to the switching bilateral filter (SBF) } \\
\text { under high densities and high commotion standard deviations. }\end{array}$ \\
\hline 3 & {$[28]$} & $\begin{array}{l}\text { Convex } \\
\text { optimisation algorithm }\end{array}$ & $\begin{array}{l}\text { They concluded that the disregarding estimations the channel furthermore, } \\
\text { remaking it utilizing Compressive Sensing, yields better comes about, than } \\
\text { the regular strategy for transmitting a picture over a channel and Denoising } \\
\text { both compressive detecting based technique and wavelet based strategy. }\end{array}$ \\
\hline 4 & {$[29]$} & $\begin{array}{l}\text { Mini-batch k-sparse dictionary } \\
\text { learning (MKDL) }\end{array}$ & $\begin{array}{l}\text { Fundamental outcomes on picture denoising have much preferable execution } \\
\text { over past lexicon learning calculations, which approves the viability of our } \\
\text { approach in meeting speed and denoising quality. }\end{array}$ \\
\hline 5 & {$[30]$} & CNN model & $\begin{array}{l}\text { Their experimental results show that the proposed CNN model can viably } \\
\text { evacuate Gaussian noise and enhance the execution of conventional picture } \\
\text { sifting strategies altogether }\end{array}$ \\
\hline 6 & {$[31]$} & $\begin{array}{l}\text { SureShrink, VisuShrink and } \\
\text { BayesShrink }\end{array}$ & $\begin{array}{l}\text { Their main purpose is to explore the wavelet coefficients results in the new } \\
\text { basis and to show the minimization achieved. }\end{array}$ \\
\hline
\end{tabular}




\section{Gaps identification}

The following gaps have been listed based on the above analysis:

1) Parametric evaluation is needed in case of prediction and quality evaluation.

2) Improvement is needed in terms of classification of image data in terms of texture and edges.

3) Data clustering is needed to cover the related pattern so that proper image construction can be achieved.

4) Inner and outer boundary value based decomposition can be helpful in noise removal.

5) Threshold based ranking can be helpful in finding the noise with the appropriate pixel position.

\section{Conclusion and future work}

Several aspects of denoising methods previously proposed have been discussed and analyzed. This paper explores and discussed the approach, advantages and future challenges based on the analysis of the presented methods. It shows the extensive way of the denoising method and the problems. In future cluster based method with proper threshold value optimization covering the inner and outer boundary values may be helpful in efficient image denoising method.

\section{Acknowledgment}

None.

\section{Conflicts of interest}

The authors have no conflicts of interest to declare.

\section{References}

[1] Shannon CE. Communication in the presence of noise. Proceedings of the IRE. 1949; 37(1):10-21.

[2] Nyquist H. Certain topics in telegraph transmission theory. Transactions of the American Institute of Electrical Engineers. 1928; 47(2):617-44.

[3] Candès EJ, Wakin MB. An introduction to compressive sampling. IEEE Signal Processing Magazine. 2008; 25(2):21-30.

[4] Ghosh P, Pandey A, Pati UC. Comparison of different feature detection techniques for image mosaicing. ACCENTS Transactions on Image Processing and Computer Vision. 2015; 1(1):1-7.

[5] Tropp JA, Gilbert AC. Signal recovery from random measurements via orthogonal matching pursuit. IEEE Transactions on information theory. 2007; 53(12):4655-66.

[6] Kumar M, Katti CP. An efficient ID-based partially blind signature scheme and application in electroniccash payment system. ACCENTS Transactions on Information Security. 2017; 2(6):36-42.
[7] Chitra AD, Ponmuthuramalingam P. Face recognition with positive and negative samples using support vector machine.2016; 2(5):16-9.

[8] Mohapatra BN, Panda PP. Histogram equalization and noise removal process for enhancement of image. ACCENTS Transactions on Image Processing and Computer Vision. 2017; 3(9): 22-5.

[9] To AC, Moore JR, Glaser SD. Wavelet denoising techniques with applications to experimental geophysical data. Signal Processing. 2009; 89(2):14460.

[10] TV NP, Hemanth VK, Kumar S, Soman KP, Soman A. Comparative study of recent compressed sensing methodologies in astronomical images. In eco-friendly computing and communication systems 2012 (pp. 108-16). Springer, Berlin, Heidelberg.

[11] Dubey S, Hasan F, Shrivastava G. A hybrid method for image denoising based on wavelet thresholding and RBF network. International Journal of Advanced computer Research. 2012; 2(4):167-72.

[12] Liua J, Shi C, Gao M. Image denoising based on BEMD and PDE. In international conference on computer research and development 2011 (pp. 110-2). IEEE.

[13] Motwani MC, Gadiya MC, Motwani RC, Harris FC. Survey of image denoising techniques. In proceedings of GSPX 2004 (pp. 27-30).

[14] Candes EJ, Tao T. Decoding by linear programming. IEEE Transactions on Information Theory. 2005; 51(12):4203-15.

[15] Singh J, Dubey RB. Reduction of noise image using LMMSE. International Journal of Advanced Computer Research. 2012; 2(5): 147-2.

[16] Anandan P, Sabeenian RS. Curvelet based image compression using support vector machine and core vector machine-a review. International Journal of Advanced Computer Research. 2014; 4(15):675-81.

[17] Veena PV, Devi GR, Sowmya V, Soman KP. Least square based image denoising using wavelet filters. Indian Journal of Science and Technology. 2016; 9(30).

[18] Lang C, Li G, Li J, Zhao X. Combined transform image denoising based on morphological component analysis. In international conference on multimedia technology 2011 (pp. 4871-4). IEEE.

[19] Su K, Fu H, Du B, Cheng H, Wang H, Zhang D. Image denoising based on learning over-complete dictionary. In international conference on fuzzy systems and knowledge discovery 2012 (pp. 395-8). IEEE.

[20] Zhang GD, Yang XH, Xu H, Lu DQ, Liu YX. Image denoising based on support vector machine. In spring congress on engineering and technology 2012 (pp. 14). IEEE

[21] Chithra K, Santhanam T. Hybrid denoising technique for suppressing Gaussian noise in medical images. In IEEE international conference on power, control, signals and instrumentation engineering 2017 (pp. 1460-3). IEEE. 
Shroff et al.

[22] Soni N, Kirar K. Transform based image denoising: a review. In international conference on recent innovations in signal processing and embedded systems 2017 (pp. 168-71). IEEE.

[23] Pang J. Improved image denoising based on Haar wavelet transform. In smartworld, ubiquitous intelligence \& computing, advanced \& trusted computed, scalable computing \& communications, cloud \& big data computing, internet of people and smart city innovation 2017. IEEE.

[24] Yang W, Liu J. Denoising fluorescence molecular image by k-means clustering. In IEEE international conference on computer and communications 2017 (pp. 1847-50). IEEE.

[25] Ankarao V, Sowmya V, Soman KP. Sparse image denoising using dictionary constructed based on least square solution. In international conference on wireless communications, signal processing and networking 2017 (pp. 1165-71). IEEE.

[26] Rubel A, Lukin V. Denoising efficiency analysis based on no-reference image quality assessment. In advanced trends in international conference on radioelecrtronics, telecommunications and computer engineering 2018 (pp. 898-902). IEEE.

[27] Rani KS, Satyanarayana RV. Image denoising using boundary discriminated switching bilateral filter with highly corrupted universal noise. In international conference on energy, communication, data analytics and soft computing 2017 (pp. 1515-21). IEEE.
[28] Devi S, Mohan P. A comparison of compressive sensing application for image denoising with wavelet denoising. In international conference on intelligent sustainable Systems 2017 (pp. 137-41). IEEE.

[29] Xue W, Zhang W. Block dictionary learning with 10 regularization and its application in image denoising. In 13th international conference on natural computation, fuzzy systems and knowledge discovery 2017 (pp. 1807-1813). IEEE.

[30] Liu Z, Yan WQ, Yang ML. Image denoising based on a CNN model. In 2018 4th international conference on control, automation and robotics 2018 Apr 20 (pp. 389-93). IEEE.

[31] Gupta V, Mahle R, Shriwas RS. Image denoising using wavelet transform method. In international conference on wireless and optical communications networks 2013 (pp. 1-4). IEEE.

[32] Ghimpețeanu G, Batard T, Bertalmío M, Levine S. A decomposition framework for image denoising algorithms. IEEE Transactions on Image Processing. 2016; 25(1):388-99.

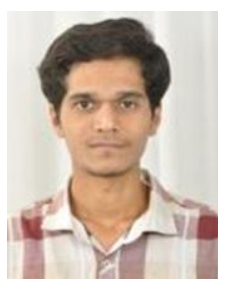

Mr. Abhishek Dipak Shroff completed B.E. in Computer Engineering from North Maharashtra University, Jalgaon in 2015 and he is currently pursuing M.tech in Computer Science and Engineering from Sri Satya Sai university of Technolgy and Medical Sciences, Sehore, MP, India.

Email: drabhishekshroff@gmail.com 